\title{
Téoros
}

Revue de recherche en tourisme

\section{Agritourisme et territoires}

Le cas du Massif central

\section{Jean-François Mamdy, Nathalie Disez et Monique Begon}

Volume 20, numéro 2, été 2001

URI : https://id.erudit.org/iderudit/1071643ar

DOI : https://doi.org/10.7202/1071643ar

Aller au sommaire du numéro

Éditeur(s)

Université du Québec à Montréal

ISSN

0712-8657 (imprimé)

1923-2705 (numérique)

Découvrir la revue

Citer cet article

Mamdy, J.-F., Disez, N. \& Begon, M. (2001). Agritourisme et territoires : le cas du Massif central. Téoros, 20(2), 44-51. https://doi.org/10.7202/1071643ar d'utilisation que vous pouvez consulter en ligne.

https://apropos.erudit.org/fr/usagers/politique-dutilisation/ 


\section{Agritourisme et territoires \\ Le cas du Massif central}

\section{Jean-François Mamdy, Nathalie Disez et Monique Begon}

Statistically, agritourism in France is still marginal. Even though tourism on the farm does not make up the largest share of rural tourism, it remains the reference. For agriculture professionals it has become a promising avenue for diversification in the last decade. For those concerned by countryside development, agritourism is an opportunity; it meets increasing demands of consumers and planners, and it helps strengthen the local economic fabric through its multiple indirect effects.

The findings of a research conducted in the Massifcentral show that the holdall term agritourism covers quite distinct activities. Certain forms of agritourism activities are purely heritage-based, whereas others have evolved as potential sources of extra income. A proportion of agritourism, albeit minor, is a business activity.

However, agritourism is still spatially diffuse. It generates no significant effects on rural demographics or employment, even at very local levels. On the other hand it helps to consolidate businesses and keep families on the land. It is often a link in local economic activity, and it strengthens the external image and identity of specific rural areas.
D lusieurs points de vue s'affrontent aujourd'hui sur la question de l'agritourisme. Pour les uns, l'agritourisme est une forme particulière de prestation touristique : le repas en ferme-auberge, la veillée en chambre d'hôte, la visite d'exploitation sont l'occasion de renouer avec une campagne préservée et imaginaire, de retrouver ses racines paysannes. De ce point de vue, il convient de porter sur le phénomène agritouristique un regard intéressé, mais prudent, dans la mesure où les aspirations du touriste sont toujours promptes à se retourner.

Pour d'autres, l'agritourisme est une opportunité de diversifier les activités agricoles. Longtemps réticentes à cette ouverture, les organisations professionnelles agricoles font aujourd'hui de l'agritourisme un cheval de bataille: « $I l$ faut que nous prenions le tourisme à sa vraie valeur: une production qui est la continuité de l'exploitation agricole» (Chezalviel, 1992). L'entrée par la production se veut volontariste : il s'agit d'agir pour le développement de l'activité agritouristique au sein de l'exploitation agricole.

Il existe encore une autre voie : celle qui considère 1'agritourisme comme l'émergence d'un nouveau modèle de développement, renouvelant le concept d'entreprise agricole et, plus généralement, la relation de l'agriculture au territoire. L'agritourisme est ici considéré comme participant d'un processus global d'aménagement et de développement rural, voire un levier de développement territorial (GREFFE, 1992). Cette troisième voie révèle une ambition, celle de l'aménage- ment du territoire et du développement local, et elle inspire l'esprit de notre réflexion.

L'objet de cet article, qui rend compte d'un travail de recherche mené de 1994 à 1996 sur le Massif central (Mamdy, 1994 ; Mamdy, 1995 ; Disez, 1996 ; ENITAC, 1995), est d'analyser les formes, les pratiques et les développements du phénomène agritouristique dans leur relation au territoire.

\section{Le concept d'agritourisme}

\section{Une confusion dans les termes}

L'expérience française du tourisme à la ferme est ancienne : le gîte rural chez l'agriculteur en a été l'archétype dans les années soixante (De Farcy et De Gunsbourg, 1967). La formule, d'abord réservée aux agriculteurs des Alpes de Haute-Provence, s'est rapidement généralisée à l'ensemble du territoire et aux ruraux. Le succès des gîtes se résume en quelques chiffres : 146 en 1955,11000 en 1970, 38000 en 1995, 43500 en 2000 (Gîtes de France dans Lobry, 1994 ; CNRTER, 1999).

Aussi dans l'esprit du public confond-on assez naturellement gîte rural, tourisme à la ferme et tourisme rural. Pourtant, les agriculteurs représentent moins de $40 \%$ des propriétaires de gîtes répertoriés à la Fédération Nationale des Gîtes de France et les gîtes ne constituent qu'une des formes d'hébergement du tourisme à la ferme et, a fortiori, du tourisme rural. Cette confusion n'est du reste pas spécifiquement française (Grolleau, 1987 ; Fiquet, 
1992). Il semble même qu'elle soit encore plus systématique chez nos voisins européens.

La notion de tourisme à la ferme doit être précisée et notamment isolée du concept de tourisme rural, auquel elle est trop souvent assimilée. Le tourisme rural concerne, en effet,

toutes les formes de tourisme en espace rural, qu'il s'agisse de tourisme à la ferme, de petite hôtellerie familiale ou de réalisations associatives ou municipales ; il évoque à la fois un tourisme d'activités, de plein air et un tourisme de découverte des milieux naturels, des cultures locales, de la gastronomie [...] (Fiquet, 1992).

\section{Les diverses conceptions de I'agritourisme}

Levons d'abord une première ambiguïté en considérant comme synonymes les expressions d'agritourisme, d'agrotourisme ou de tourisme à la ferme. Une analyse sémantique permettrait sans doute de marquer quelques nuances, en référence aux logiques qui animent ceux qui les emploient.

J.-A. Martins-Cruz (1995) définit l'agritourisme comme «le tourisme à la ferme proposé par des agriculteurs, et allié à leurs activités traditionnelles $»$. Plus précis, G. Bazin (1993) enrichit la définition et l'explicite par ses diverses prestations : «l'agritourisme correspond à des activités d'accueil, d'hébergement, de restauration et de loisirs, dont le siège est l'exploitation agricole ». A. Hardt (1994) confirme le panorama des activités, mais souligne avec force la finalité du projet agritouristique : l'agritourisme concerne « toute activité d'accueil, d'hébergement et de fourniture de services à des tiers, utilisant les ressources d'une exploitation agricole, dans le but de contribuer à sa viabilité économique et à la sauvegarde de son rôle productif et plurifonctionnel ». Cette dernière définition a le mérite de la clarté car elle précise les tenants et aboutissants du projet agritouristique : l'avenir de l'ex-

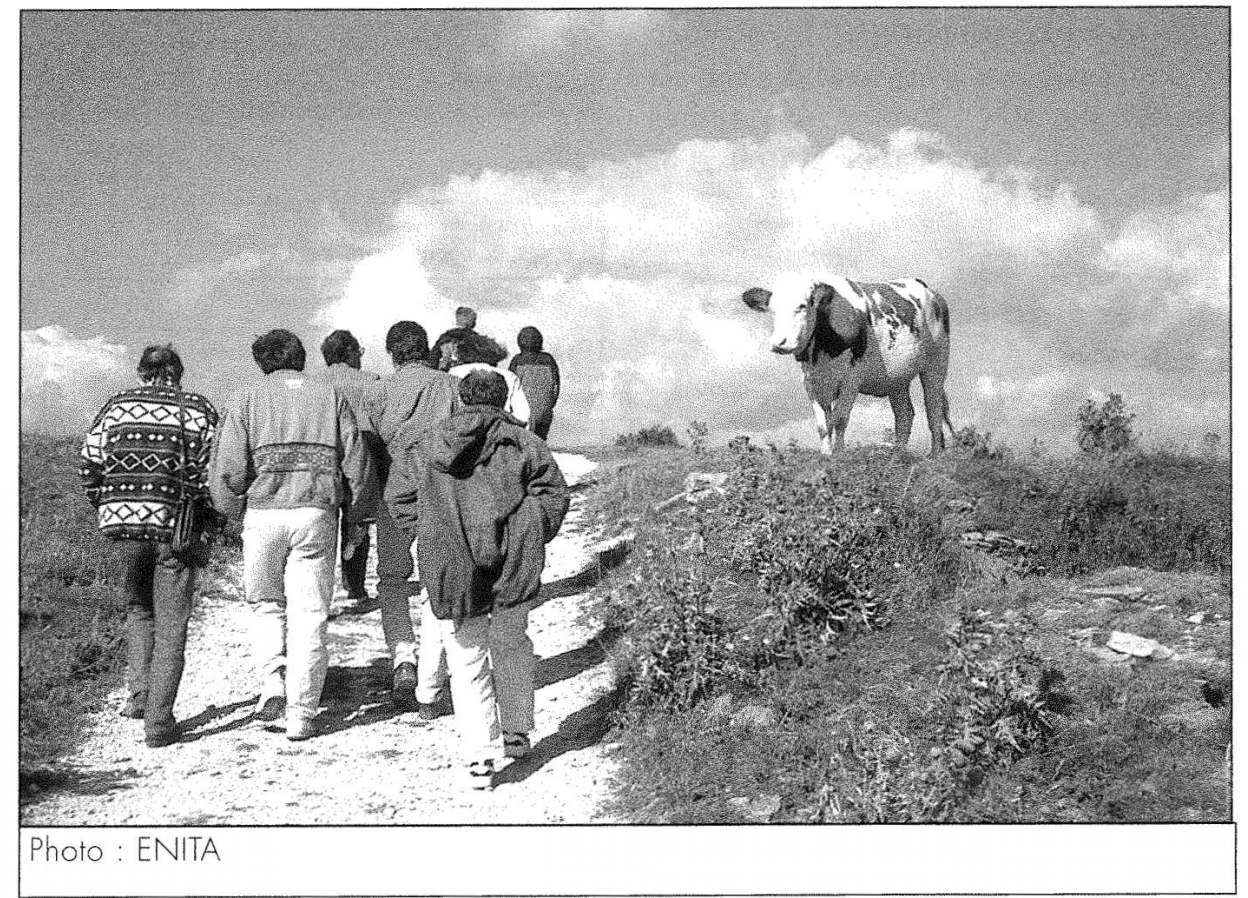

ploitation agricole. Mais, dans cet esprit, il faut remarquer que tout agriculteur non reconnu juridiquement comme exploitant agricole ne relève plus de l'agritourisme. Ainsi, l'agriculteur retraité ne pourrait pas offrir de prestations agritouristiques ! Arguant de ce fait, $\mathrm{H}$. Grolleau considère que « la notion d'agritourisme ne doit pas correspondre à un statut, mais à un type de prestation » (Grolleau et Ramus, 1986). Plus généralement l'agritourisme peut être défini dans sa dimension culturelle : «l'activité agritouristique vise la valorisation des produits agricoles et la diffusion de la connaissance de la civilisation rurale au travers des moyens touristiques» (Francario et Paolini, 1991 dans MartinsCruz, 1991). Au-delà de l'exploitation ou de la prestation, c'est l'homme, le paysan "personnage symbole de la culture d'autrefois et de l'enracinement dans le terroir, objet du rapport ambigu entre tradition et modernité » (Alphandery et coll., 1989), qui constituerait le fondement du phénomène agritouristique.

La spécificité de l'agritourisme ne seraitelle pas en définitive contenue dans la relation singulière que le prestataire, l'agriculteur établit par prestation interposée avec le touriste, son hôte et client? Le débat sur le concept d'agritourisme n'est donc pas clos. Considère-t-on l'avenir de l'exploitation agricole ou la spécificité de la prestation agritouristique ? Place-t-on en priorité le point de vue du producteur et du revenu agritouristique, celui du consommateur, ou celui du développeur? Ces choix ne sont pas sans conséquences sur l'approche du phénomène agritouristique, la prise en compte et la mesure qui s'ensuivent. Pour notre part, nous privilégierons l'entrée par la production et les producteurs, dans une perspective de développement territorial.

\section{L'agritourisme : formes et évolutions dans le Massif central}

\section{État des lieux quantitatif et spatial}

Parler du phénomène agritouristique suppose bien entendu un relevé d'observations le plus complet possible, d'où le choix d'inventorier les réalités du terrain avec un souci d'exhaustivité, à une échelle compatible avec l'observation de détails, mais suffisamment large pour être signifiante. Le choix du Massif central, vaste ensemble de moyennes montagnes (500 à $1500 \mathrm{~m}$ ) situé au centre de la France, couvrant 7,5 millions d'hectares, soit environ $15 \%$ du territoire national, nous a paru pertinent pour observer et analyser 
le phénomène agritouristique. Il s'est donc agi de dresser un état des lieux des exploitations et des formes d'activités agritouristiques en Massif central pour l'année 1995.

\section{Méthode de repérage}

\section{Limite des sources statistiques}

Les statistiques du tourisme ignorent les réalités agritouristiques, les statistiques agricoles 1'abordent à la marge. Conçu pour estimer la production agricole française, le Recensement Général de l'Agriculture (RGA), principale source de données agricoles, apporte des renseignements peu précis concernant les productions agritouristiques, considérées comme annexes. Ainsi en France, au dernier recensement de 1988, 15080 exploitations agricoles, soit 1,4\% de l'ensemble des structures françaises, proposaient une prestation d'hébergement ; soit une progression nette de 2383 unités par rapport à 1979 , où elles ne représentaient que $1 \%$ de l'ensemble des exploitations. Cependant, ces données sont anciennes (la collecte d'information dans le cadre du RGA se déroulant au cours de l'hiver 2000-2001) et ne prenant pas en compte l'activité de restauration, ni les formes d'animation, minimisant ainsi l'ensemble agritouristique.

L'accès aux fichiers informatiques des statistiques agricoles permet d'affiner la collecte d'information. En 1988, quatre rubriques concernant les « activités liées au tourisme » ont été insérées dans le questionnaire du RGA : restauration seule, hébergement seul, restauration et hébergement, ni restauration ni hébergement. C'est à partir de ces données que l'Institut National d'Études et de Recherche sur la Montagne de Grenoble (INERM) s'est intéressé aux " activités touristiques des ménages agricoles » (Malavieille et Tardif, 1993) en axant son analyse sur les zones de montagne ou défavorisées, où l'on observe une concentration des exploitations agritouristiques supérieure à la moyenne française $(2,3 \%$ contre $1,4 \%)$. Plus récemment, Danièle Capt (1994) a approfondi l'analyse des données du RGA

\begin{tabular}{|c|c|}
\hline \multicolumn{2}{|c|}{ 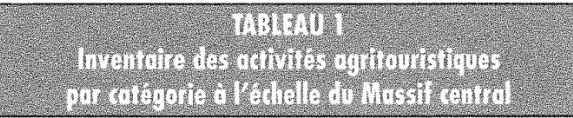 } \\
\hline $\begin{array}{l}\text { Type } \\
\text { de prestations }\end{array}$ & $\begin{array}{c}\text { Nombre } \\
\text { de prestations }\end{array}$ \\
\hline $\begin{array}{l}\text { Gîtes (rural, de groupe, } \\
\text { d'étape, de pêche, équestre) }\end{array}$ & 1448 \\
\hline Chambres d'hôtes ou paysannes & 438 \\
\hline Campings à la ferme ou aires naturelles & 332 \\
\hline $\begin{array}{l}\text { Vente directe associée à une autre } \\
\text { activité }\end{array}$ & 311 \\
\hline Tables d'hôtes ou tables paysannes & 272 \\
\hline $\begin{array}{l}\text { Fermes-auberges ou auberges } \\
\text { à la ferme, repas à la ferme }\end{array}$ & 205 \\
\hline $\begin{array}{l}\text { Accueil d'enfants (à la journée) ou } \\
\text { gîtes d'enfants }\end{array}$ & 103 \\
\hline Fermes équestres & 98 \\
\hline $\begin{array}{l}\text { Autres (goûters, randonnées, } \\
\text { fermes de séjour...) }\end{array}$ & 311 \\
\hline Nombre total de prestations & 3518 \\
\hline
\end{tabular}

Source: États des lieux, ENITAC, 1995

à l'échelle de la France. Il reste cependant difficile de dresser un profil particulier de l'entreprise agritouristique.

\section{Une approche pragmatique de I'agritourisme}

Ce constat de faiblesse dans l'appareil statistique conduit donc à retenir le principe d'un repérage direct des activités agritouristiques. Mais, pour y parvenir, il faut définir au préalable ce que l'on entend par exploitant et activité agritouristique.

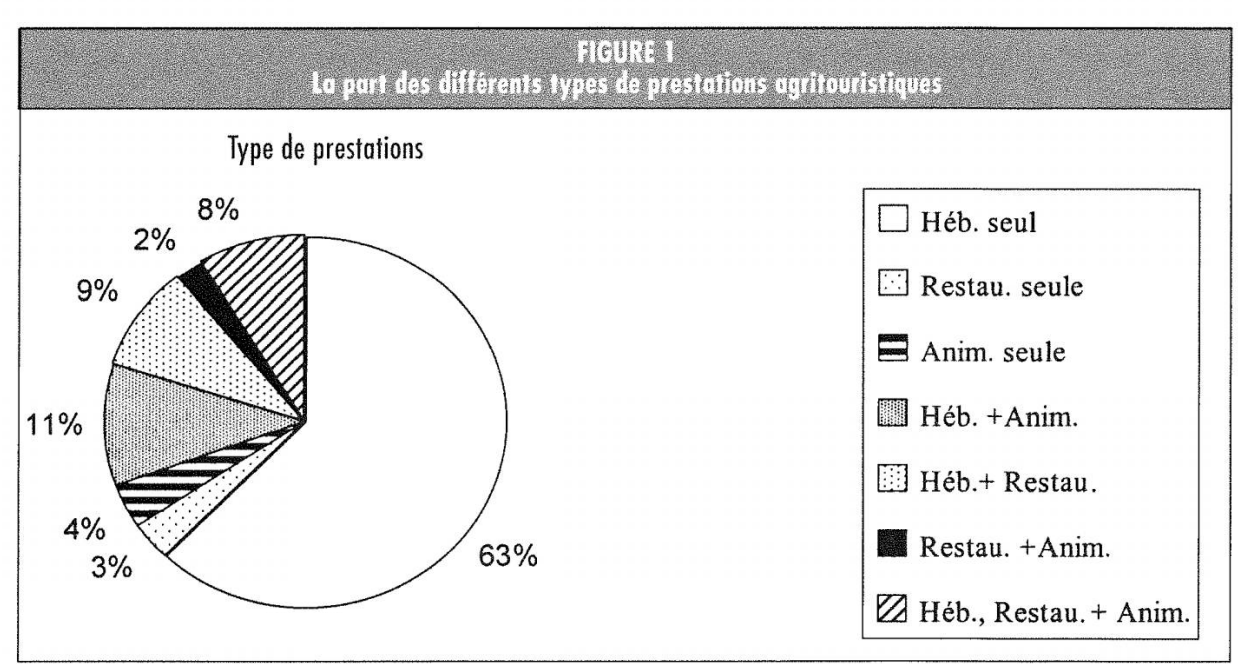

Source: États des lieux, ENITAC, 1995.
Pour repérer les exploitants, la collaboration des Mutualités Sociales Agricoles s'avère précieuse. En contrepartie, l'inventaire constitué ne comprend que des agriculteurs travaillant la moitié de la surface minimale d'installation ou des agriculteurs retraités. Ont été prises en compte les activités réalisées par l'exploitant, qu'il soit en activité ou en retraite, ou plus largement par sa famille, en général son conjoint.

Quant aux activités qui ne relèvent pas directement de l'activité agricole et ne «prolongent » pas l'exploitation, elles ont été exclues du champ de l'agritourisme. La vente directe de produits fermiers soulève une autre question. Est-ce une forme touristique? Si la vente est destinée à une clientèle de proximité, il semble abusif de parler d'activité touristique. Par contre, si elle est destinée au touriste, l'activité peut être considérée comme touristique. La vente directe de produits fermiers n'a donc été prise en considération, que si elle était associée à une autre activité touristique.

En ce qui concerne l'inventaire des activités agritouristiques, trois sources principales ont été utilisées : les guides départementaux «Bienvenue à la ferme », les documents promotionnels des « Gîtes de France » et différentes brochures émanant des Comités départementaux du tourisme, des Offices de tourisme ou de l'association « Accueil Paysan ». 


\section{Importance et répartition des activités agritouristiques}

Agriculteurs et types de prestations

À l'échelle du Massif central, on dénombre 2261 agriculteurs et 3518 prestations (voir tableau 1).

Retenons en première analyse la diversité des formes d'activités agritouristiques, avec cependant une nette prépondérance du gîte qui représente $41 \%$ de l'ensemble des prestations. Cependant, ce type d'observation comptable ne rend pas compte des combinaisons de prestations sur une même exploitation. Aussi, les prestations ont-elles été réunies en trois catégories selon leur nature :

- hébergement : gîtes rural, d'étape, de groupe, chambres d'hôtes, campings,

- restauration : repas à la ferme, auberges et tables d'hôtes,

- animation : goûters à la ferme, fermes et relais équestres, visites de fermes...

Certaines appellations comme les fermes de séjours ou les gîtes d'enfants regroupent à la fois les trois natures d'activités. La figure 1 montre la part des différents types de prestations agritouristiques.

Il reste que l'hébergement (seul ou associé à une autre activité) est la prestation la plus courante : il concerne $91 \%$ des exploitations ayant une activité d'accueil : $22 \%$ des prestataires proposent une forme de restauration à la ferme et $25 \%$ une activité d'animation. Par ailleurs, $30 \%$ des agriculteurs proposent des prestations combinées associant hébergement, restauration ou animation.

\section{Répartition des activités agritouristiques dans l'espace}

\section{Une répartition différenciée}

Les activités agritouristiques sont présentes sur l'ensemble du Massif central (voir figure 2). Cependant l'agritourisme, considéré en nombre d'exploitants agritouristiques, est plus diffus dans le nord du Massif (Creuse, Puy de Dôme, Loire et Allier). Dans le sud-ouest (Lot, Corrèze) et dans la partie orientale du Massif, dans

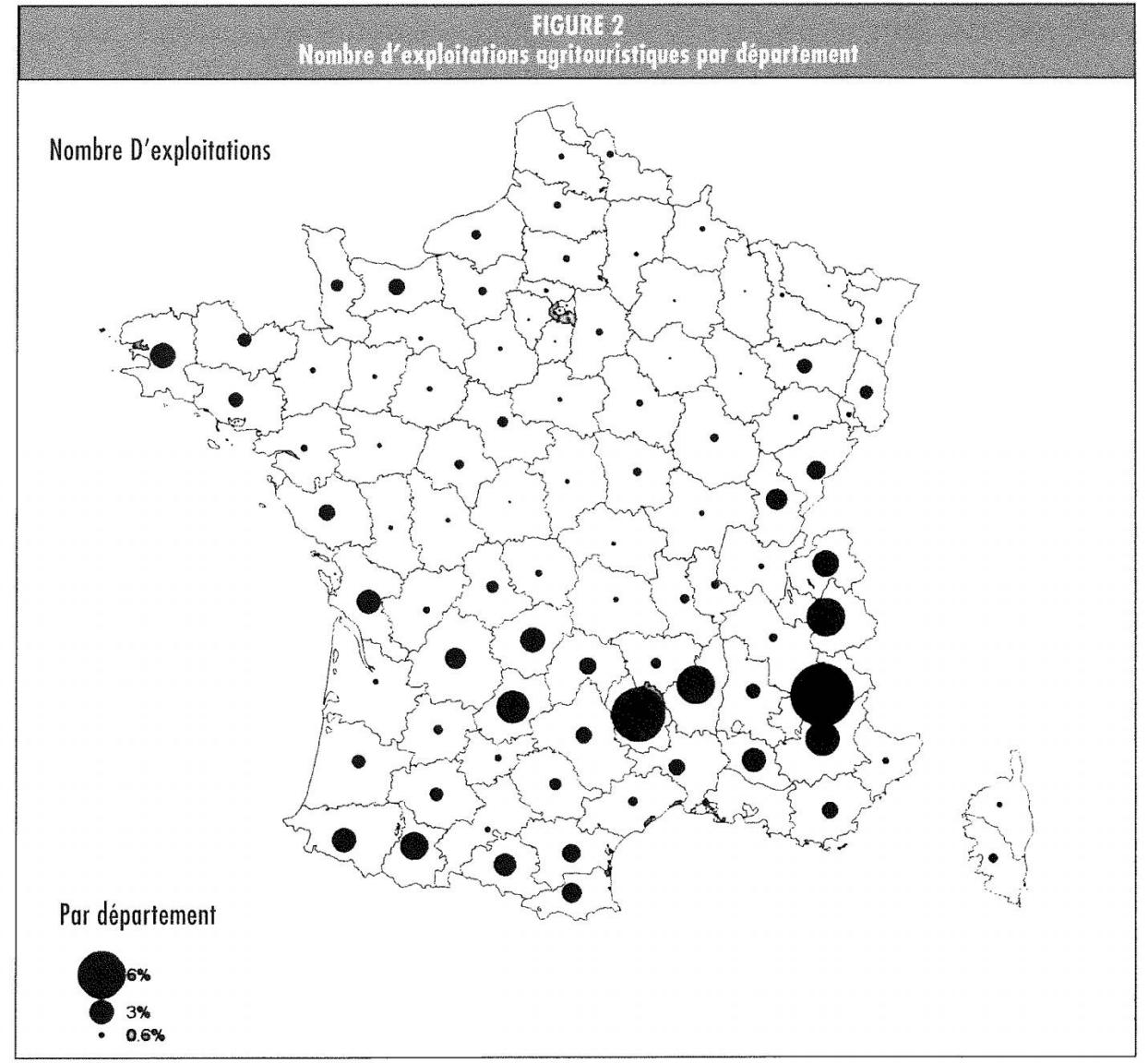

TRAME/FNGEDA, Clermont-Ferrant, 20-21 octobre 1993.

une moindre mesure, le tourisme à la ferme est une pratique courante.

Rapportée au nombre d'exploitations, la proportion d'exploitations agritouristiques est de l'ordre de 2,2 \% (Enquête de structures 1993 dans De Caix, 1995), mais la proportion fluctue beaucoup d'un département à l'autre: moins de $1 \%$ dans l'Aveyron à plus de $5 \%$ dans le Lot et la Lozère.

\section{Les types de prestations}

Les types de prestations varient selon les départements, même si la base reste l'hébergement. Plusieurs départements se singularisent par un type de prestation : les fermes-auberges ou l'accueil d'enfants en Haute-Loire, le camping et les fermes équestres en Ardèche, notamment. La place des activités d'animation est importante en Corrèze, Cantal et Haute-Loire. La Creuse, enfin, se démarque par son peu d'équipements. Les chambres d'hôtes, dont la création est très récente, se répartissent sur l'ensemble du Massif central.

Dans le Lot, $98 \%$ des exploitants proposent une prestation d'hébergement, unique ou combinée à une autre activité. A l'opposé, dans le département du Puy-de-Dôme, les prestataires d'hébergement ne représentent que $65 \%$ des exploitants agritouristiques. Les départements de l'Ardèche, de la Loire et de la Haute-Loire offrent des activités agritouristiques diversifiées.

Certains agriculteurs combinent plusieurs activités de nature différente : hébergement et restauration, restauration et animation, hébergement et animation ou les trois à la fois. Les plus fortes proportions de prestataires associant des prestations de nature différente se trouvent dans les départements de l'est du Massif central (Loire et Ardèche).

L'inventaire par département à l'échelle du Massif fait donc apparaître d'importantes concentrations d'activités dans certains secteurs géographiques et des formes 
différenciées d'activités par zone. D’ores et déjà des hypothèses peuvent être formulées pour rendre compte ici ou là des particularités territoriales. Relevons l'isolement géographique de certains espaces, défavorable au développement agritouristique ; a contrario, la proximité de grands bassins de population, propice aux courts séjours. Le caractère déjà touristique de certaines zones ainsi que la solidité ou la fragilité de l'agriculture régionale peuvent aussi influencer l'expansion du tourisme à la ferme. Ces quelques pistes de réflexion seront approfondies par l'étude localisée.

L'état des lieux présenté demeure cependant statique. Il ne permet pas de comprendre réellement le développement des expériences agritouristiques dans le temps. Une observation très fine des expériences et des pratiques s'impose.

\section{Les dynamiques agritouristiques}

L'étude des dynamiques agritouristiques s'appuie sur un échantillon d'une soixantaine d'exploitations agritouristiques du Massif central, sélectionnées lors des journées «Tourisme vert et agriculture ${ }^{1}$ ».

\section{Des exploitations très différenciées}

La caractéristique première de la population d'exploitations agritouristiques est la diversité des systèmes de production. Un tiers des exploitations combine des spéculations traditionnelles (vaches allaitantes, laitières ou ovins viande) à l'activité touristique, mais les deux tiers se dispersent en multiples productions (vingt activités de productions agricoles différentes) associant agriculture et tourisme.

Les surfaces d'exploitation s'échelonnent de la très petite exploitation de moins de dix hectares à la grande structure de plus de cent hectares (un cinquième de l'échantillon); les capacités de main-d'œuvre varient selon les exploitations, mais sont relativement élevées, presque systématiquement supérieures à deux U.T.A. (Unité de Travail Annuel). De même, la nature et la place de l'atelier touristique changent d'une expérience à l'autre. Ainsi une agricultrice du Cantal anime la visite d'un buron, sommairement aménagé ; elle raconte l'histoire des paysans et la vie des troupeaux et des hommes sur la Planèze. La restauration du buron n'a pas nécessité de grands investissements, l'organisation des animations sur rendez-vous mobilise peu de temps. À l'opposé, un jeune couple d'agriculteurs du Forez a équipé dans un corps de ferme un gîte d'étape. Il propose à la fois la table d'hôte et la fermeauberge et réalise à l'occasion des animations musicales. L'activité touristique prend dans ces conditions la place principale sur l'exploitation, apporte l'essentiel des revenus, mobilisant de fait des capacités d'investissement considérables (plus de un million de francs dans une exploitation sur sept).

\section{Un système évolutif et innovant}

La dynamique de projet constitue une autre caractéristique forte de notre population agritouristique (elle concerne les quatre cinquièmes de l'échantillon) : les agriculteurs souhaitent explicitement développer encore leur activité touristique, en termes de croissance quantitative et de qualité de prestation. Leur stratégie s'oriente massivement vers une diversification accrue, d'où une complexification du système en place.

L'observation des trajectoires d'exploitation confirme d'ailleurs cette voie de développement. Par exemple, un couple d'agriculteurs de la région de Saint-Flour décide en 1976 d'ouvrir un camping à la ferme. Trois ans plus tard, sollicité par la clientèle qui ne trouve pas à se restaurer à proximité, le couple ouvre une petite ferme-auberge d'une capacité de quinze couverts, puis en 1982 il installe quatre chambres d'hôtes. Incités par le désir de leur fille de rester sur l'exploitation, ils agrandissent la ferme-auberge (55 couverts) et créent deux nouvelles chambres « 3 épis » en 1992. En 1993, les exploitants aménagent une salle de séjour pour les hôtes.

À l'évidence, le développement de l'activité agritouristique procède par étapes successives, avec prudence, mise à l'essai, puis confirmation. Il y a là un véritable processus d'apprentissage pour l'agriculteur confronté au « mal connu », à l'expérimental, à l'innovant. Par bien des aspects, l'innovation est au cœur de la dynamique du projet agritouristique. II s'agit d'abord d'une innovation de produits : nouveaux produits fermiers à caractère gastronomique, médicinaux, artistiques. Il s'agit encore d'innovations dans l'organisation, soit individuelle (l'art de gérer la complexité d'un système à activités multiples), soit collective (pour produire, transformer, ou commercialiser). Signalons la ferme-auberge d'Ally, en Haute-Loire, qu'anime depuis vingt ans une dizaine de familles d'agriculteurs, ou le « Circuit gourmand des gentianes » dans la haute vallée de la Cère, dans le Cantal, qui réunit des agriculteurs - hébergeurs et restaurateurs - sur les thèmes de la gastronomie et de la convivialité. Il s'agit aussi d'innovations commerciales : rompant avec la tradition des prix agricoles garantis, les producteurs agritouristiques ont adopté la démarche marketing en étudiant leur marché, leur clientèle, leur communication, leur mode de distribution et de vente.

\section{Le choix de l'agritourisme}

Le dynamisme, la diversité, l'originalité de l'expérience agritouristique permettentelles d'esquisser le profil des acteurs ?

L'observation de la population conduit à distinguer deux groupes d'exploitants en fonction de leur origine professionnelle ou géographique. Les chefs d'exploitation d'origines agricole et locale représentent les trois quarts de la population. Leur motivation tient à la fois de l'attente d'un revenu et de l'intérêt d'une activité d'accueil pour rompre l'isolement social et géographique. Les femmes sont souvent à l'origine de l'initiative touristique et la gèrent au quotidien. L'introduction de l'activité agritouristique s'effectue quand l'exploitation atteint une phase de croisière (au bout d'une dizaine d'années). Les chefs d'exploitation nouvellement installés dans l'agriculture, n'ayant pas de précédent professionnel agricole, constituent le deuxième groupe. Ce sont en quelque sorte des néo-ruraux. Ils peuvent être éventuellement du pays, mais sont le plus souvent 
originaires d'ailleurs. Leur motivation est principalement un choix de vie, une passion pour l'activité.

Il ne faudrait cependant pas négliger les conditions « objectives » qui ont favorisé l'essor de l'agritourisme depuis quelques années: d'abord la mise en place des quotas laitiers en 1984 et la vague des mesures qui ont suivi, limitant les productions, puis le « retournement» de la profession agricole sur la question de la diversification et de l'agritourisme, enfin la mise en place d'une politique européenne d'appui aux projets touristiques, complétée par des mesures d'aide des collectivités territoriales. Ainsi le phénomène agritouristique marque un élan significatif depuis la fin de la décennie quatre-vingts. Sur l'ensemble des exploitations étudiées, quelques expériences seulement remontent aux années 1970. De 1975 à 1985, les créations se sont amplifiées, mais le décollage de l'activité se situe en Massif central à partir de 1988 (70\% des exploitations enquêtées) et se poursuit depuis.

\section{Trois logiques de fonctionnement}

L'explication du succès de l'agritourisme, du développement significatif de l'offre, ne peut cependant tenir dans un schéma simpliste associant acteurs volontaires et contexte favorable. L'analyse de ces différentes expériences montre que les motivations et les stratégies s'ordonnent selon trois logiques :

\section{- Une logique domestique et patrimoniale.} L'activité touristique est un alibi pour sauvegarder le patrimoine familial. Dans cet esprit, l'investissement financier peut être important, sans souci pour autant de rentabilité économique. On investira 300000 F pour restaurer un gîte dont les recettes annuelles s'élèveront à $10000 \mathrm{~F}$.

- Une logique de complément de revenu. L'activité agricole reste prépondérante et le tourisme marginal. Peu exigeante en main-d'œuvre, l'activité touristique est peu rémunératrice. On rencontre cette situation sur des exploitations moyennes de 2 U.T.A. Les chambres d'hôtes,

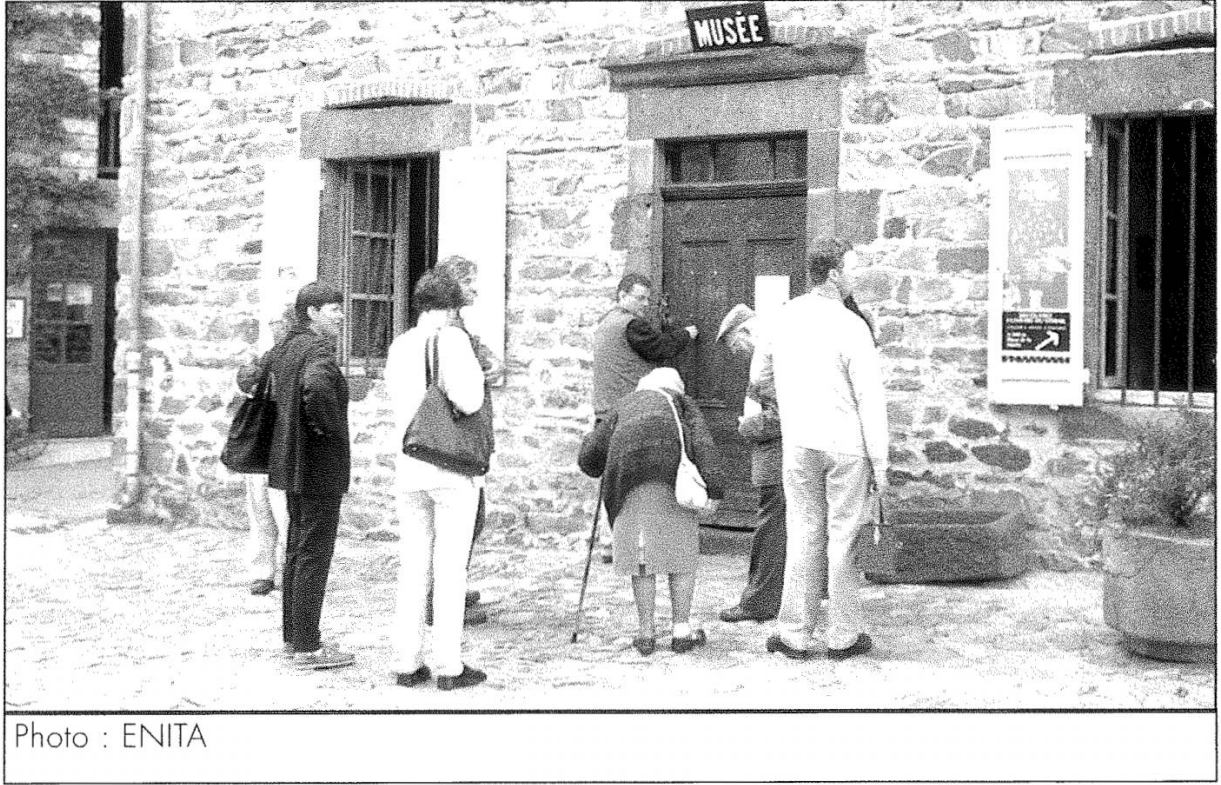

en petit nombre, illustrent cette pratique. L'initiative et la gestion sont souvent l'affaire des femmes qui attendent de cette activité une certaine responsabilité et des contacts.

- Une logique entrepreneuriale. L'activité touristique devient importante, voire prépondérante dans l'exploitation. Elle correspond à une motivation pour l'accueil et à un souci de rentabilité économique et financière. Elle s'accompagne d'une qualification, d'un comportement professionnel. Cependant, deux situations peuvent être distinguées. La première correspond à une juxtaposition des activités agricole et touristique. Chaque activité fonctionne indépendamment l'une de l'autre, dans des cadres juridiques souvent distincts (GAEC - Groupement Agricole d'Exploitation en Commun - et SARL - Société Anonyme à Responsabilité Limitée - par exemple). Il s'agit alors de grandes structures (plus de 100 hectares) fortement pourvues en main-d'œuvre (3 à 4 U.T.A.). La seconde présente des activités très intégrées. L'activité touristique commande la production agricole : l'entreprise fonctionne selon une logique commerciale. On valorise au mieux les produits agricoles par la transformation et la vente directe, la restauration et l'hébergement associés. Il s'agit alors de petites structures très intégrées souvent tenues par de néo-ruraux.
Il serait cependant abusif de cloisonner les différentes logiques. Un même exploitant peut au cours de sa carrière passer de l'une à l'autre : une stratégie de complément de revenu pour essayer, puis d'entreprise pour se développer, et finalement le repli, à l'approche de la retraite, sur les valeurs patrimoniales. Appréhendé globalement à l'échelle d'un grand espace comme le Massif central ou, ponctuellement, sous l'angle de la réalisation particulière, l'agritourisme apparaît sous le signe d'une extrême diversité de formes, d'expériences, de processus.

Il reste cependant que deux éléments exercent une influence déterminante dans le développement de l'agritourisme : la spécificité du territoire et la stratégie des agriculteurs. Pourrait-on dès lors mieux comprendre le phénomène en étudiant de près la relation entre territoire et stratégie d'acteur? Dans quelle mesure l'agritourisme est-il déterminé par le territoire où il se situe et, réciproquement, comment l'activité transforme-t-elle le territoire, le développe-t-elle en s'y inscrivant?

\section{Agritourisme et terrictoires}

L'analyse statistique et cartographique de l'état des lieux de l'agritourisme en Massif central met en évidence une localisation diffuse des entreprises, avec cependant une tendance à la polarisation près des sites 
touristiques ou des grandes agglomérations urbaines. Elle souligne selon les départements des concentrations inégales, des pratiques différenciées, des caractères spécifiques attachés, semble-t-il, aux modes d'organisation agricoles, touristiques, territoriaux. Pour mieux comprendre, il faut désormais changer d'échelle. C'est en effet à l'échelle locale que se dessine la spécificité des terroirs.

Six territoires ont été retenus sur la base de « pays » organisés en vue du développement. Ils ont en commun une forte densité agritouristique (de $3 \%$ à $7 \%$ d'exploitants agritouristiques selon les territoires), mais se différencient par la localisation géographique et le mode d'organisation et de développement agritouristiques. Il s'agit des Monts du Forez versant Loire, du Mézenc-Meygal en Haute-Loire, du «pays touristique cévenol » entre Gard et Lozère, du « pays de Salers » entre la Dordogne et les Monts du Cantal, de «Monts et Barrages » en Haute-Vienne et de la partie lotoise de la vallée de la Dordogne.

Notre propos est maintenant d'expliquer en quoi la spécificité d'une localisation ou d'une configuration organisationnelle sur un territoire rend compte du développement du tourisme à la ferme.

\section{Diversité des contextes selon les territoires}

Dans les six territoires, l'agriculture connaît un déclin plus ou moins marqué. Certains espaces sont encore dominés par une agriculture traditionnelle relativement solide (Salers, Monts et Barrages, vallée de la Dordogne), d'autres connaissent une agriculture très affaiblie (Monts du Forez), voire totalement marginalisée (pays cévenol, Mézenc-Meygal).

Pays touristiques ? Trois d'entre eux bénéficient d'une bonne notoriété touristique, l'un parce qu'il englobe le site de Salers (cité médiévale de caractère), un autre du fait de son rattachement à la région des Cévennes qui jouit d'une grande renommée touristique. La vallée de la Dordogne, avec les sites de Rocamadour et du gouffre de Padirac, est sans contexte le territoire le plus touristique. En l'absence de grande notoriété, des territoires comme le Forez ou le Mézenc tirent cependant profit des clientèles de proximité.

En Haute-Vienne et dans les Cévennes, la structuration territoriale (pays d'accueil) est clairement orientée vers le tourisme et joue un rôle actif en faveur de son développement, mais les dissensions internes pour le premier territoire et la jeunesse pour l'autre ne leur permettent pas de s'engager à fond. Dans les Monts du Forez, l'association touristique «Espace Lignon Forez » tient une place éminente dans la dynamique de développement. Dans les autres territoires, par contre, la Chambre d'Agriculture fédère et organise le développement. Le Mézenc-Meygal présente une très forte participation des acteurs agritouristiques, soutenus par la Chambre d'Agriculture. Ce sont souvent des personnes jeunes qui ont cherché à structurer l'agritourisme au sein du territoire. Les nombreux néo-ruraux installés dans le pays cévenol ont aussi joué un rôle important dans le développement de l'agritourisme et ils ont dessiné une nouvelle orientation du métier d'agriculteur dans ce territoire.

\section{Des stratégies d'organisation différenciées}

L'absence de structuration est la caractéristique dominante de l'agritourisme, quel que soit le territoire considéré. Les prestataires se considèrent avant tout comme des agriculteurs. Il existe cependant quelques actions communes qui organisent l'agritourisme au sein du territoire.

\section{Des actions communes, mais pas d'organisation aboutie}

À l'exception de la vallée de la Dordogne lotoise, où n'existe aucune organisation territoriale des producteurs agritouristiques, dans les autres régions, diverses formes de structuration, sur le plan de la commercialisation notamment, ont vu le jour. Ainsi, Al Païs, point de vente collectif de Salers, est né et subsiste grâce à la volonté et à la ténacité de la conjointe d'un exploitant. Après un premier échec, le point de vente s'est implanté au sein d'une exploitation et parvient à dégager des revenus suffisants pour rémunérer les producteurs (11 agriculteurs) et un vendeur à mi-temps. Mais l'engagement collectif reste très faible. De même, l'Association Vienne-Maulde, regroupement de conjointes d'exploitants en Haute-Vienne, organise la vente de produits fermiers et des prestations de services (buffets campagnards). Elle a permis dans de nombreux villages de revitaliser ou de recréer des marchés de pays. L'association, très attachée au territoire, ne souhaite cependant pas s'agrandir par l'adhésion de nouveaux membres, par crainte d'une perte d'identité. Terroir-Cévennes, point de vente collectif installé à St Jean du Gard (pays cévenol) témoigne quant à lui d'une démarche dynamique et d'une volonté de cohésion de la part des adhérents (20 agriculteurs et 12 artisans). La démarche est ici professionnelle, tant dans l'organisation de la production que dans le fonctionnement du point de vente : mise en place d'un contrôle de qualité et d'un comité d'agrément pour les nouveaux produits, étude des clientèles. Les associés voudraient aujourd'hui évoluer vers une maison de pays. De même, après des débuts hésitants, 1'Association des «Fermiers du Mézenc » a connu un développement exponentiel et offre maintenant un point de vente collectif et une auberge.

Nécessité fait loi, les initiatives d'organisation collective existent. Cependant, les expériences les plus abouties relèvent des terroirs où l'agriculture est en grande difficulté (Cévennes, Mézenc). Les promoteurs de ces actions communes sont essentiellement des jeunes, souvent néoruraux. Dans les pays où l'agriculture est solide et continue de structurer l'économie rurale, les initiatives collectives en agritourisme restent cependant très marginales.

\section{Des logiques différentes selon les pays}

Interrogés sur leur motivation, leur pratique et leur projet de développement, les agriculteurs se répartissent, selon les zones, en fonction de logiques dominantes. Il 
semble que sur des territoires marqués par une production agricole traditionnelle, où le revenu agricole n'est pas trop faible (Monts et Barrages, Salers), la recherche de rentabilité économique ne soit pas le souci prioritaire. Par contre, dans les Cévennes ou le Mézenc-Meygal, on trouve des agriculteurs jeunes, entreprenants, qui cherchent à développer une activité touristique rentable et rémunératrice. Dans les Cévennes, le statut agricole peut constituer le meilleur moyen pour créer l'activité touristique ; dans le Mézenc-Meygal, c'est l'activité touristique qui peut permettre d'assurer la survie de l'exploitation agricole. A contrario, dans la vallée de la Dordogne, la stratégie dominante est patrimoniale : les agriculteurs s'investissent très peu dans le tourisme et proposent des prestations peu élaborées (location de gîte). On voit donc que la logique professionnelle peut s'inscrire dans des objectifs différents à long terme et que la réalisation de ceux-ci peut infléchir l'avenir de l'exploitation agricole.

À l'évidence, il existe bien des «logiques de territoire » au sens où un ensemble de conditions naturelles, de traditions économiques et sociales, influence les pratiques collectives et individuelles des acteurs de l'agritourisme. Cependant, au stade actuel de développement du processus et compte tenu du faible niveau d'organisation territoriale, les stratégies individuelles d'acteurs dessinent sur chaque pays le visage de la diversité plutôt que celui d'une réelle spécificité territoriale de l'agritourisme.

\section{Conclusion}

Les enseignements de la recherche conduite sur le Massif central invitent d'abord à différencier les expériences que l'on rassemble trop facilement sous le même terme d'agritourisme. Certaines formes d'activité agritouristique relèvent d'une démarche purement patrimoniale, d'autres formes correspondent à la recherche d'un revenu complémentaire. Pour une partie, certes minoritaire, l'agritourisme est une activité d'entreprise. Les entrepreneurs ruraux qui dirigent les exploitations agritouristiques sont porteurs de dyna- misme et de changement dans le monde rural.

Cependant, l'agritourisme reste un phénomène diffus dans l'espace. L'organisation collective des producteurs reste problématique et incertaine dans la durée. Ainsi la dispersion, la diversité, la difficulté d'organisation créent-elles des limites au développement du processus et à ses répercussions sur les territoires. L'agritourisme n'engendre pas d'effets significatifs sur l'occupation de l'espace ou la création d'emploi, même à l'échelle micro-régionale. Par contre il contribue à la consolidation des entreprises, au main-

\section{Bibliographie}

Alphandery, P., P. Bitoun, et Y. Dupont (1989), Les Champs du départ, Paris, 265 p.

Bazin, G. (1993), Le développement de l'agritourisme en France, Rencontres Internationales de FEZ sur le Développement des zones défavorisées, Paris, INRA, 14 p.

Capt, D. (1994), Demande de biens différenciés, comportements spatiaux et diversification de l'activité des exploitations agricoles, ENESAD/INRA, Dijon, 366 p. et annexes.

Chezalviel, M., (1992), «Le tourisme, un produit agricole parmi d'autres », Gazette du Tourisme, 2 mars, n' 1087 , p. 9.

CNRTER (Centre National de Ressources du Tourisme en Espace Rural) (1999), Tourisme en espace rural, acteurs, clients, produits, Dossier de synthèse $n^{\circ} 29$, mars, 102 .

De Caix, Camille (1995), « Rumeurs agricoles », Agreste, Cahier n² 22, juin, p. 15-19.

De Farcy, H., et P. De Gunsbourg (1967), Tourisme en milieu rural, Paris, Flammarion, $219 \mathrm{p}$.

Disez, N. (1996), Agritourisme et développement territorial, Thèse de Doctorat ès Géographie, Université de Clermont-Ferrand, 241 p. et annexes.

Disez N. (1999), « Agritourisme, logiques d'acteurs ou logiques de territoires ", Économie Rurale, $\mathrm{n}^{\circ} 250$, p. 40-46.

ENITAC (1995), Contribution de l'Agritourisme au développement rural, Rapport final, novembre, $34 \mathrm{p}$.

ENITAC/SORBONNE (1995), Actes du Colloque Agritourisme et Développement Local, 7 juin, $140 \mathrm{p}$.

Fiquet, A.-M. (1992), Le tourisme rural et la C.É.E., Espaces, n 114, février-mars, p. 4-8. tien des familles sur place ; il participe souvent des dynamiques locales et conduit à renforcer l'image externe et l'identité des terroirs. Produit d'une démarche d'acteur, pionnière et individuelle, l'agritourisme ne sauvera pas le monde rural, mais il constitue un élément-clé du renouveau économique et culturel des pays ruraux.

\section{Jean-François Mamdy est profes- seur, Nathalie Disez et Monique Begon sont maîtres de conférence à l'École Nationale d'Ingénieurs des Tra- vaux Agricoles de Clermont-Ferrand, site de Marmilhat, 63370 LEMPDES, France.}

Greffe, X. (1992), « Tourisme rural, développement économique et emploi », Initiatives Locales de création d'emplois, Paris, O.C.D.E., p. 131-161.

Grolleau, H. (1987), Le tourisme rural dans les douze États membres de la Communauté Économique Européenne, T.E.R., décembre, 96 p.

Grolleau, H., et A. Ramus (1986), Espace rural, espace touristique : le tourisme à la campagne et les conditions de son développement en France, Paris, La Documentation française, $381 \mathrm{p}$.

Hardt, A. (1994), Directeur du Centre Européen pour la Formation Agricole et Rurale, CEPFAR, Bruxelles, Éditorial de la Lettre de l'AFRAT, janvier, $\mathrm{n}^{\circ} 36$.

Lobry, Jean (1994), Le printemps du tourisme vert - acteurs et témoins - 1945/1970, TER, août, $86 \mathrm{p}$.

Malavieille, D., et P. Tardif (1993), Activités touristiques des ménages agricoles, CEMAGREF-INERM, Étude n²50, 46 p et annexes.

Mamdy, J.-F. (1994), Le Développement du tourisme à la ferme, nouvelles pratiques, nouvelles questions, nouvelles problématiques, Colloque de l'Association des Ruralistes français, 24-25 novembre, $7 \mathrm{p}$.

Mamdy, J.-F. (1995), Agritourisme, levier de développement local, Actes du Colloque Agritourisme et Développement local, ParisSorbonne, 7 juin, p. 41-46.

Martins-Cruz, J.-A. (1991), L'agrotourisme dans la C.E.E. : Utopie ou réalité ? Euratour, Bruxelles, $137 \mathrm{p}$. 